\title{
A New Interpretation to Groszkowski's Effect
}

\author{
Mostafa Jafari Nokandi \\ Faculty of Information Technology \\ and Electrical Engineering \\ University of Oulu \\ Oulu, Finland \\ mostafa.jafarinokandi@oulu.fi
}

\author{
Timo Rahkonen \\ Faculty of Information Technology \\ and Electrical Engineering \\ University of Oulu \\ Oulu, Finland \\ timo.rahkonen@oulu.fi
}

\author{
Sumit Pratap Singh \\ Faculty of Information Technology \\ and Electrical Engineering \\ University of Oulu \\ Oulu, Finland \\ sumit.singh@oulu.fi
}

\author{
Aarno Pärssinen \\ Faculty of Information Technology \\ and Electrical Engineering \\ University of Oulu \\ Oulu, Finland \\ aarno.parssinen@oulu.fi
}

\begin{abstract}
This paper presents an alternative interpretation to Groszkowski's effect, which states that the oscillation frequency of a harmonic oscillator is shifted due to the higher harmonic currents flowing into the LC tank. Groszkowki's analysis starts by taking a strictly memoryless amplifier, to which he derives and expression for balance of reactive powers. Then he interprets the frequency shift as a matter of balance of reactive energy in the tank. Here we show that although a conductive amplifier is memoryless, it can still show phase shift at the fundamental tone. This is due to mixing of the fundamental and harmonics in the nonlinear i-v response of the amplifier. Now the new interpretation comes from the oscillation condition: if the amplifier shows phase shift, the tank needs to compensate it, and this causes the shift in the oscillation frequency. The mechanism is explained, analysed symbolically, and verified numerically.
\end{abstract}

Index Terms-AM-PM, Groszkowski's effect, Oscillator, Nonlinear system, Frequency pulling

\section{INTRODUCTION}

In 1933, Groszkowski noticed that when the nonlinearity of the oscillator circuit increases, its center frequency shifts. As a result of this finding, he presented an analysis to study the effect of the harmonics caused by a nonlinear negative resistor on the fundamental frequency of the tank circuit [1]. Based on his analysis he assumes that there must be a balance of total energy in the capacitance and in the inductance, and hence the increased harmonic power must force the fundamental frequency downwards.

Another noteworthy phenomenon that occurs in all nonlinear circuits is AM-to-PM conversion, which denotes a phase shift caused by changes in the amplitude of a signal applied to the nonlinear element. It is a common source of distortion in power amplifiers, and in circuits like oscillators where phase is of utmost importance and gain is a function of amplitude [2], AM-to-PM conversion can have serious consequences [3], e.g. increasing the phase noise and undesired frequency pulling. Several papers in literature have studied the influences of this phenomenon on circuits using Volterra analysis or harmonic balance simulations, such as [4]. It has surveyed AM-to$\mathrm{AM}$ and AM-to-PM conversions in an LDMOS RF power amplifier, and also illustrated the effect of harmonic voltages on AM-PM.

The energy balance approach has been accepted as an interpretation of the Groskowszki's effect. In this paper, an alternate interpretation to Groszkowski's effect is presented. First, it is shown that when a memoryless non-linear amplifier is driven by the fundamental tone and sum of harmonics, the harmonic-to-harmonic mixing causes amplitude-dependent phase shift (AM-PM) into the fundamental tone. From this and the zero-phase oscillation condition, it is concluded that the frequency deviation of the tank circuit is a direct consequence of AM-PM phenomenon, which is in turn caused by the nonlinear element in the circuit.

\section{GROSZKOWSKI'S ANALYSIS}

Groszkowski's finding was that when the harmonic distortion in an oscillator increases, the oscillation frequency shifts. To analyse this effect, he used strictly behavioral and symbolic calculus, considering of a memoryless, non-linear negative resistance and a resonant circuit that can include all the reactive components of a practical amplifier circuit, resulting in the circuit in Fig. 1. As the amplifier part does not contain any energy-storing circuit elements, the system's impulse response will be $\sum_{1}^{n} a_{n} \delta^{n}(t)$. So, the instantaneous current will be a function of the voltage at that instant, leading to (1) over one cycle of the fundamental tone:

$$
\oint i d v=0
$$

On the basis of this fundamental equation, Groszkowski modeled both the input voltage $v$ and output current $i$ as sums of harmonics. Solving (1) gives a second equation

$$
\sum_{1}^{\infty} k\left|V_{k} I_{k}\right|_{i m}=0
$$




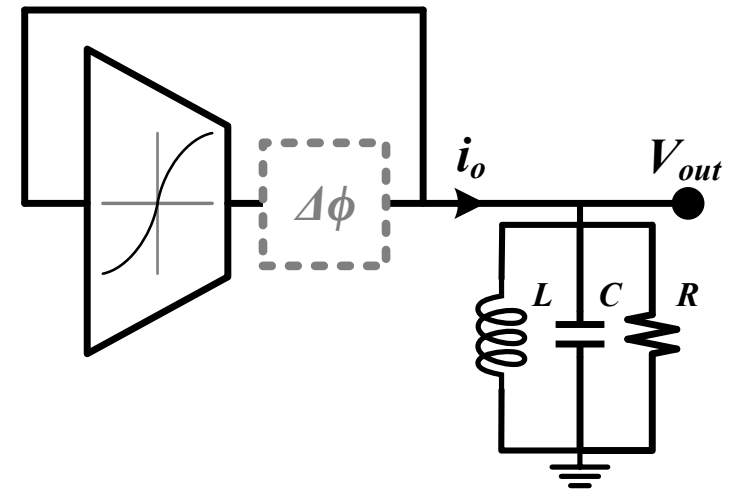

Fig. 1. Representation of the effect of AM-PM conversion in the oscillator with the consideration of phase shift (neglected by Groszkowski).

which forces reactive powers at all harmonics to cancel out. At the fundamental frequency, the voltage and current $V_{1}$ and $I_{1}$ are in-phase. However, at the harmonics, the resonator is offtune, and there is an almost 90-degree phase shift between $V_{k}$ and $I_{k}$. This means that the power at the harmonics is mostly reactive, and it can be concluded from (2) that the fundamental would also need to have a cancelling imaginary part. This has been interpreted as an energy balance: reactive power at the harmonics needs to be cancelled at the corresponding imaginary power at the fundamental tone. To achieve that, the fundamental tone needs to shift out of the resonance frequency.

\section{ANALYSING THE AM-PM IN A MEMORYLESS AMPLIFIER}

When talking about the reasons of AM-PM distortion (amplitude-dependent phase shift) in amplifiers, two reasons are commonly mentioned. First, some of the circuit capacitance can be non-linear, or the Miller capacitance may appear to be signal-dependent due to compressing gain [5](Fig. 2).

To study the typical oscillator circuit, we follow Groszkowski's approach and use a strictly memoryless negative resistance amplifier as shown in Fig. 1. We then show that it can still generate AM-PM when excited by a sum of harmonic signals. Let us start by modeling the I-V response of

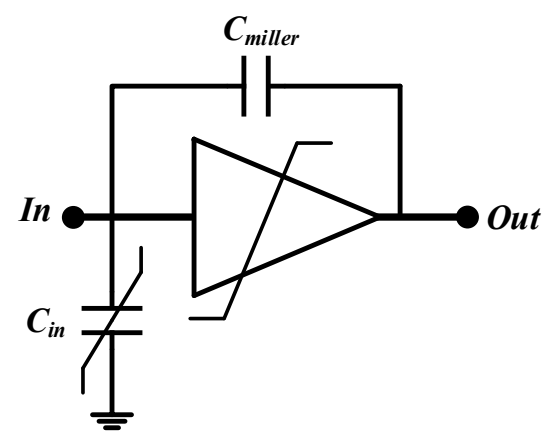

Fig. 2. Voltage dependent capacitor and gain as often mentioned sources of AM-PM . the non-linear negative resistance by a cubic polynomial (3)

$$
i=K_{1} V+K_{2} V^{2}+K_{3} V^{3}
$$

where $V$ is the total input voltage (including all the harmonics), and $K_{1}-K_{3}$ are the coefficients of the polynomial non-linear model. In a steady state condition, the fundamental, second and third harmonic voltages $V_{1}, V_{2}$ and $V_{3}$, respectively, are fed to the input of the nonlinear amplifier. So, the net input voltage $V$ of the nonlinear amplifier can be written as (4):

$$
\begin{aligned}
V= & V_{1}+V_{2}+V_{3}=a_{1} \cos \left(\omega_{o} t+\phi_{1}\right) \\
& +a_{2} \cos \left(2 \omega_{o} t+\phi_{2}\right)+a_{3} \cos \left(3 \omega_{o} t+\phi_{3}\right)
\end{aligned}
$$

After substituting (4) into (3) and neglecting those resulting terms which correspond to the harmonics above the third, we have a total fundamental output current given in (5)(seen on the top of the next page). It is important to note that this current consists of several mixing results between the input harmonics. If these harmonics have phase shift, the phase of the fundamental will also rotate.

In the following analysis, we consider one of the most common cases, the differential topology. The balanced structure cancels the even order non-linearities, so that $K_{2}=0$ and $a_{2}=0$ in (5). Hence, from (6), we can calculate the phase shift $(\Delta \phi)$ for a balanced circuit structure in (7). For comparison, the similar result for a single-ended topology with $2^{\text {nd }}$-order curvature has been analyzed in the appendix.

$$
\Delta \phi=\tan ^{-1}\left(\frac{\frac{3}{4} K_{3} a_{1} a_{3} \sin \phi_{3}}{K_{1}+K_{3}\left(\frac{3}{4} a_{1}^{2}+\frac{3}{2} a_{3}^{2}\right)+\frac{3}{4} K_{3} a_{1}^{2} a_{3} \cos \phi_{3}}\right)
$$

It can be observed from (7) that, due to the harmonics mixing in the nonlinear component, $2^{\text {nd }}$ and $3^{\text {rd }}$ harmonics are down-converted to $\omega_{o}$ as shown in Fig. 3. Due to this effect, AM-PM distortion is generated even in a completely memoryless amplifier.

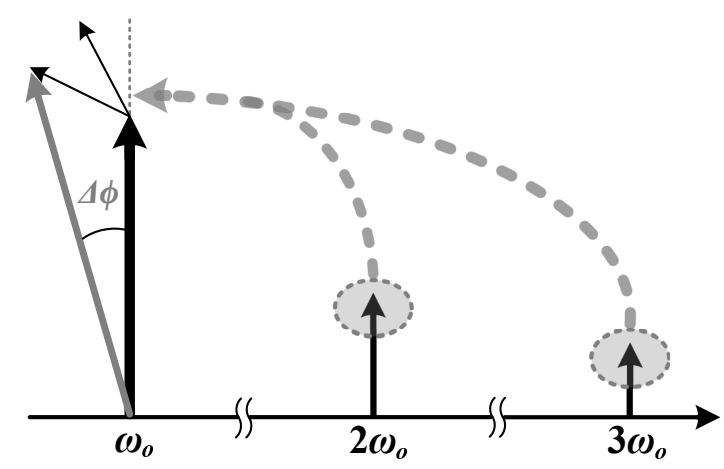

Fig. 3. Generation of AM-PM as a result of mixing from harmonic frequencies. 


$$
\begin{aligned}
\left.i_{o}\right|_{\omega_{o}}=\left(K_{1} a_{1}+K_{3}\left(\frac{3}{4} a_{1}^{2}+\frac{3}{2} a_{1} a_{2}^{2}+\frac{3}{2} a_{1} a_{3}^{2}\right)\right) \cos \left(\omega_{o} t+\phi_{1}\right)+K_{2} a_{1} a_{2} \cos \left(\omega_{o}+\phi_{2}-\phi_{1}\right) \\
+K_{2} a_{2} a_{3} \cos \left(\omega_{o} t+\phi_{3}-\phi_{2}\right)+\frac{3}{4} K_{3} a_{1}^{2} a_{3} \cos \left(\omega_{o} t+\phi_{3}-2 \phi_{1}\right)+\frac{3}{4} K_{3} a_{2}^{2} a_{3} \cos \left(\omega_{o}+2 \phi_{2}-\phi_{3}\right) \\
i_{o}=\left(K_{1} a_{1}+K_{3}\left(\frac{3}{4} a_{1}^{2}+\frac{3}{2} a_{1} a_{3}^{2}\right)\right) \cos \left(\omega_{o} t\right)+\frac{3}{4} K_{3} a_{1}^{2} a_{3} \cos \left(\omega_{o} t+\phi_{3}\right) \\
=A \cos \left(\omega_{o} t+\tan ^{-1}\left(\frac{\frac{3}{4} K_{3} a_{1} a_{3} \sin \phi_{3}}{K_{1}+K_{3}\left(\frac{3}{4} a_{1}^{2}+\frac{3}{2} a_{3}^{2}\right)+\frac{3}{4} K_{3} a_{1}^{2} a_{3} \cos \phi_{3}}\right)\right)
\end{aligned}
$$

\section{NUMERICAL VERIFICATION}

To verify the generation of the phase shift in the amplifier part, a simple MATLAB code was written where the polynomial, memoryless amplifier was driven with a sum of fundamental and harmonic input voltages. The output, corresponding to the output current was calculated and its spectral components were extracted using FFT. To eliminate the problems caused by spectral leakage, the sample duration was chosen to be an exact integer multiple of the cycle time of the fundamental tone.

The results of this analysis are shown in Fig. 4. Here the polynomial coefficients of the amplifier are $K_{1}=1.3, K_{2}=0$, $K_{3}=-0.2$, and it is driven by a fundamental tone with an amplitude of $a_{1}=1.84$, and a third harmonic with the amplitude $a_{3}=0.2$. In Fig. 3, the phase of the $3^{\text {rd }}$ harmonic is swept from -90 to +90 degrees, and the phase of the output fundamental tone is plotted. As seen, injecting the $3^{\text {rd }}$ harmonic in about -90 degree phase shift (the phase of the $3^{\text {rd }}$ harmonic impedance in a high- $Q$ LC tank) causes a fundamental phase shift of about +2 degrees.

In short, we see a small phase shift in the fundamental output when the input consists of the fundamental and phase shifted harmonics. This is in line with Groskowski's analysis $\left(\sum_{1}^{\infty} k\left|V_{k} I_{k}\right|_{i m}=0\right)$ : imaginary parts in the harmonics need to be cancelled by an imaginary part in the fundamental tone. Yet, equation (2) is not really a property of the tank, but derived from (1), which only defines that the amplifier is memoryless.

\section{Calculation of Frequency Shift $\Delta \omega$}

Based on the Barkhausen oscillation criteria, the total phase shift around the loop must be an integer multiple of $2 \pi$, meaning that the imaginary impedances of the amplifier and the resonator must cancel. As an example, it is well known that input impedance of a Pierce crystal oscillator amplifier part is capacitive. So the oscillation pulls the crystal below its inherent resonance frequency, where the impedance of the resonator is inductive - This way, the grounded caps in the Pierce oscillator pull the oscillation frequency downwards [6].

Here, the analysis assumes that the amplifier is memoryless, but it still has phase shift due to the down-converted harmonics. Therefore, the tank must generate a phase shift to compensate the phase alterations as shown in Fig. 5. Eventually, this phase shift forces the tank frequency to change from its fundamental frequency $\left(\omega_{0}\right)$ [7]. The frequency shift $(\Delta \omega)$ in the tank can be obtained from (8).

$$
\Delta \omega=\frac{\omega_{o}}{2 Q} \Delta \phi
$$

It is worth noting that the oscillator must be tuned near the center of the resonance for (9) to hold. After substituting (7) into (8), the frequency shift in the tank is obtained for the

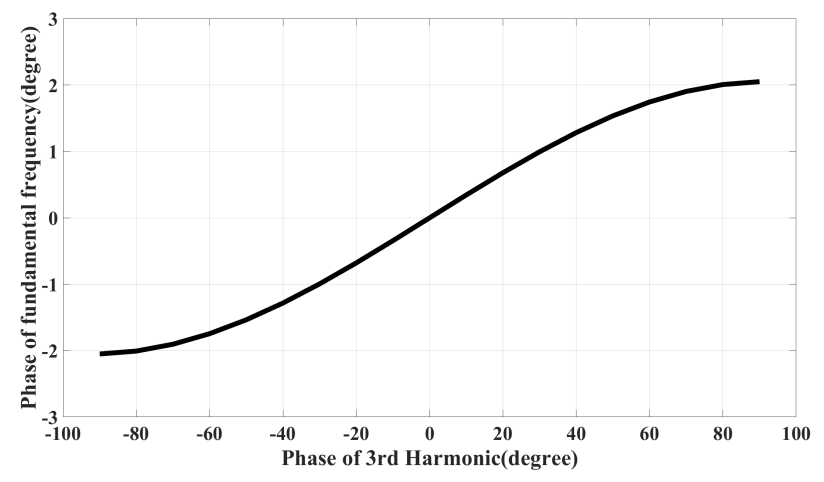

Fig. 4. Fundamental tone phase shift caused by third harmonic.

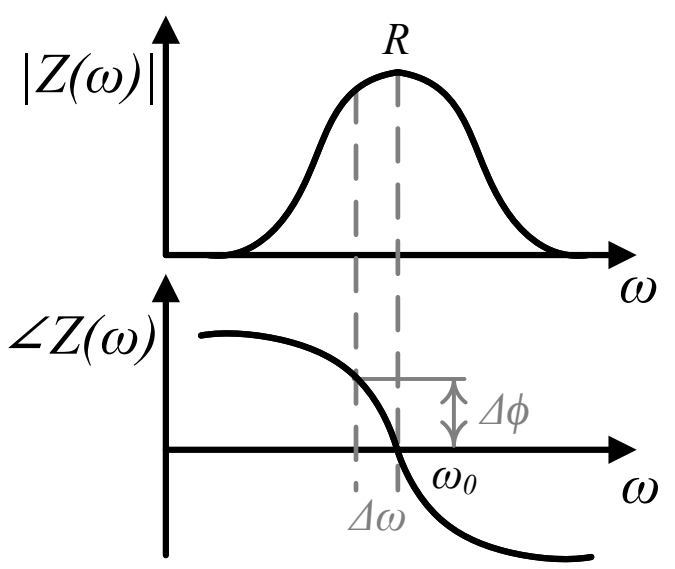

Fig. 5. Amplitude and phase spectrum of tank impedance. 


$$
\begin{aligned}
& \Delta \phi=\tan ^{-1}\left(\frac{\frac{\sin \left[\tan ^{-} 1\left(\frac{K_{2} a_{1} a_{2} \sin \phi_{2}}{K_{1} a_{1}+K_{2} a_{1} a_{2} \cos \phi_{2}}\right)\right]}{\sqrt{K_{1}^{2} a_{1}^{2}+K_{2}^{2} a_{1}^{2} a_{2}+2 K_{1} K_{2} a_{1}^{2} a_{2} \cos \phi_{2}}}+K_{3} a_{2} a_{3} \sin \left(\phi_{3}-\phi_{2}\right)}{\frac{\cos \left[\tan ^{-1} 1\left(\frac{K_{2} a_{1} a_{2} \sin \phi_{2}}{K_{1} a_{2}+K_{2} a_{1} a_{2} \cos \phi_{2}}\right)\right]}{\sqrt{K_{1}^{2} a_{1}^{2}+K_{2}^{2} a_{1}^{2} a_{2}+2 K_{1} K_{2} a_{1}^{2} a_{2} \cos \phi_{2}}}+K_{3} a_{2} a_{3} \cos \left(\phi_{3}-\phi_{2}\right)}\right) \\
& \Delta \omega=\frac{\omega_{o}}{2 Q} \tan ^{-1}\left(\frac{\frac{\sin \left[\tan ^{-} 1\left(\frac{K_{2} a_{1} a_{2} \sin \phi_{2}}{K_{1} a_{1}+K_{2} a_{1} a_{2} \cos \phi_{2}}\right)\right]}{\sqrt{K_{1}^{2} a_{1}^{2}+K_{2}^{2} a_{1}^{2} a_{2}+2 K_{1} K_{2} a_{1}^{2} a_{2} \cos \phi_{2}}+K_{3} a_{2} a_{3} \sin \left(\phi_{3}-\phi_{2}\right)}}{\frac{\cos \left[\tan ^{-} 1\left(\frac{K_{2} a_{1} a_{2} \sin \phi_{2}}{K_{1} a_{1}+K_{2} a_{1} a_{2} \cos \phi_{2}}\right)\right]}{\sqrt{K_{1}^{2} a_{1}^{2}+K_{2}^{2} a_{1}^{2} a_{2}+2 K_{1} K_{2} a_{1}^{2} a_{2} \cos \phi_{2}}}+K_{3} a_{2} a_{3} \cos \left(\phi_{3}-\phi_{2}\right)}\right)
\end{aligned}
$$

differential topology in (9)

$\Delta \omega=\frac{\omega_{o}}{2 Q} \tan ^{-1}\left(\frac{\frac{3}{4} K_{3} a_{1} a_{3} \sin \phi_{3}}{K_{1}+K_{3}\left(\frac{3}{4} a_{1}^{2}+\frac{3}{2} a_{3}^{2}\right)+\frac{3}{4} K_{3} a_{1}^{2} a_{3} \cos \phi_{3}}\right)$

\section{DISCUSSION}

In this paper, Groszkowski's effect is analyzed from a new point of view. Groszkowski's reactive power balance equation, shown in (2), is often considered as a built-in energy-balance property of a resonator circuit while it is actually derived from the properties (1) of a memoryless amplifier. However, in this paper, we found out that a memoryless amplifier driven by a sum of phase shifted harmonics can still show phase shift at the fundamental frequency. Combining the phase shift in the amplifier with the Barkhausen's requirement of 0 degree phase shift in the loop, we obtain an alternative interpretation for the frequency shift: it is the AM-PM conversion of the amplifier that is causing the reactive pulling of the tank. This interpretation is not limited to the energy balance, but applies also e.g. to an amplifier with harmonic injection, where this effect could be used as a method of impedance tuning.

The formulization of the frequency offset caused by a memoryless nonlinear element producing second and third order harmonics paves the way for dealing with nonlinearity more effectively in a variety of circuits including power amplifiers, oscillators and frequency multipliers. For instance, the phase shift can be adjusted to achieve the ideal value by tuning the ratios of the harmonics to the fundamental signal in phase shifters.

Finally, let us illustrate a modification to the circuit level improvements proposed by Groszkowski. Groszkowski claimed that frequency shift can be minimized by a) running the oscillator in a linear mode, b) filtering the harmonics, or c) equalizing the imaginary energy. The first two make perfect sense: when the input voltage of the amplifier has no harmonics, it can not generate any phase shift, so the loop delay is not changed. To take the third approach, Groszkowski proposes adding a small inductor in series with the tank capacitance to balance the reactive energy. However, it both attenuates and phase shifts the harmonics, making the induced AM-PM in the amplifier smaller, and hence reducing the frequency pulling. A similar effect could also be achieved by adding a series reactance to the amplifier to cancel its imaginary part, which has been used e.g. to force crystal oscillators to run at the exact resonance frequency of the crystal.

\section{ACKNOWLEDGEMENT}

This research has been financially supported by the Academy of Finland 6Genesis Flagship (grant 318927).

\section{REFERENCES}

[1] J. Groszkowski, "The Interdependence of Frequency Variation and Harmonic Content, and the Problem of Constant-Frequency Oscillators," in Proceedings of the Institute of Radio Engineers, vol. 21, no. 7, pp. 958-981, July. 1933.

[2] E. Vidal, A. Poveda and M. Ismail, "Describing functions and oscillators," in IEEE Circuits and Devices Magazine, vol. 17, no. 6, pp. 7-11, Nov. 2001.

[3] Maas, Stephen A. Nonlinear Microwave and Rf Circuits. 2nd ed. Boston (Mass.): Artech House, 2003.

[4] J. P. Aikio and T. Rahkonen, "A Comprehensive Analysis of AMAM and AMPM Conversion in an LDMOS RF Power Amplifier," in IEEE Transactions on Microwave Theory and Techniques, vol. 57, no. 2, pp. 262-270, Feb. 2009.

[5] T. Rahkonen, S. Hietakangas and J. Aikio, "AM-PM distortion caused by transistor's signal-dependent input impedance," 2011 20th European Conference on Circuit Theory and Design (ECCTD), pp. 833-836, Linkoping, Aug. 2011.

[6] E. A. Vittoz, M. G. R. Degrauwe and S. Bitz, "High-performance crystal oscillator circuits: theory and application," in IEEE Journal of SolidState Circuits, vol. 23, no. 3, pp. 774-783, June 1988.

[7] B. Razavi, "A study of phase noise in CMOS oscillators," in IEEE Journal of Solid-State Circuits, vol. 31, no. 3, pp. 331-343, March. 1996.

\section{APPENDIX}

The analysis of the single-ended topology can be done in the same way as the differential case. Similarly, the output current and phase shift $(\Delta \phi)$ corresponding to the single-ended topology are approximated by condition $\left(K_{3}=0, a_{3}=0\right)$ and given in (10) and (11) respectively.

$$
\begin{aligned}
i_{o}= & K_{1} a_{1} \cos \left(\omega_{o} t\right)+K_{2} a_{1} a_{2} \cos \left(\omega_{o} t+\phi 2\right) \\
& +K_{3} a_{2} a_{3} \cos \left(\omega_{o} t+\phi_{3}-\phi_{2}\right)
\end{aligned}
$$

Similarly, $\Delta \omega$ is obtained for the single ended topology by substituting (11) into (8) shown in (12). 